\title{
Comparative Study of Embodied Energy in Different Walling Materials
}

\author{
Udawattha C.D.* and Halwatura R.U. \\ Department of Civil Engineering, University of Moratuwa, Sri Lanka \\ *udawatthe@gmail.com
}

\begin{abstract}
Calculating embodied energy of a construction material is a wholistic method of measuring the environmental sustainability factor because by reducing embodied energy can mitigate the environmental pollution. Along resolution, the embodied energy is the amount of all the energy devoured while manufacturing transporting constructing and maintaining specific product. Sri Lanka is using brick and cement block as main walling material. Brick walling materials use $1,5541 \mathrm{MJ}$ of energy per $100 \mathrm{ft}^{2}$ and cement block using $1,1542.9 \mathrm{MJ}$ per $100 \mathrm{ft}^{2}$. In this study, the embedded energy of brick and other mostly available walling materials such as cement blocks, wattle and daub, mud concrete block, rammed earth wooden planks and steel sheets were accounted and compared according to the origin of energy consumption. The processes of walling material were carefully studied and analyze to calculate embodied energy. One 100 $\mathrm{ft}^{2}$ square $(10 \times 10 \mathrm{ft})$ was used to measure and compare the embodied energy content. Then overall rank was given considering the embedded energy cost and usability factor.

According to the collected data, the most industrious walling materials such as brick and cement blocks consuming large amount of energy in the manufacturing process. Wattle and daub is the obvious eco-friendly walling material. Since the study compare walling material, overall soil blocks made of cement and soil (altering wattle and daub) shows best results with minimum embedded energy and yet provide essential strength to support the structure. Notwithstanding, replacing brick and cement block with alter walling material would save millions of trees.
\end{abstract}

Keywords: Embodied Energy, Energy content, Process analysis, Walling materials 